\title{
Quantitative Characterization of Microscopic Variations in the Cross-Link Density of Gels
}

\author{
G. E. Mitchell,,,† L. R. Wilson, ${ }^{\ddagger}$ M. T. Dineen, ${ }^{\ddagger}$ S. G. Urquhart, ${ }^{\S, \#}$ F. Hayes, ${ }^{\dagger}$ \\ E. G. Rightor ${ }^{\dagger}$ A. P. Hitchcock, ${ }^{\perp}$ and H. Ade ${ }^{*, \S}$ \\ Analytical Sciences Laboratory, The Dow Chemical Company, 1897 Building, \\ Midland, Michigan 48667; Superabsorbent Products R\&D, The Dow Chemical Company, \\ 1603 Building, Midland, Michigan 48667; Department of Physics, North Carolina State University, \\ Raleigh, North Carolina 27695; and Brockhouse Institute for Materials Research, \\ McMaster University, Hamilton, Ontario L8S 4M 1, Canada
}

Received May 14, 2001; Revised Manuscript Received September 24, 2001

\begin{abstract}
We report the visualization and quantitative analysis of the cross-link structure in model core/shell hydrogel polymers on the microscopic scale, in a fully swollen state, using soft X-ray microscopy. The cross-link density in these materials and their microscopic or even nanoscopic variation critically influence materials characteristics, yet the cross-link density is difficult to characterize by conventional methods. By the use of soft X-ray microscopy, one can investigate these materials in a fully swollen state and thus directly visualize and quantitatively determine the cross-link structure on a microscopic scale. Materials that were cross-linked by different methods were shown to give rise to differently shaped profiles. Abrupt and gradient cross-link density profiles have been investigated, and the spatial variation in their cross-link density has been determined quantitatively.
\end{abstract}

\section{Introduction}

Gels and networks and gel-forming materials are common in nature (e.g., in-vivo actin and myosin filament networks, agars, and pectins) as well as in synthetic materials (e.g., hydrogels for hygiene applications, drug delivery, ion-exchange resins, and network polymers such as unsaturated polyester resins). ${ }^{1-4}$ Gels in particular are a spectacular form of soft matter. They are neither solid nor liquids, or conversely both solid and liquid, and respond to very weak external perturbations: They can swell or contract in response to minute changes of salinity, $\mathrm{pH}$, or temperature. One exciting future application making use of a response to an external stimuli is the use of gels for artificial muscles. ${ }^{2}$ A critical feature of gels (and polymer networks) that influences and determines many material properties is the cross-link density. F or example, the rate of release of pharmaceuticals, pesticides, insecticides, pheromones, and fertilizer from polymer matrixes may be controlled by the cross-link density and the response of the gel and network to some externally originating stimuli. ${ }^{3}$ To prepare optimized cross-link microstructures and to understand fundamental characteristics of synthetic and natural gels, it is essential to have quantitative measurements of the cross-link density, including variations on a submicron spatial scale. Despite its importance, the determination of the cross-link density in polymer gels and networks at high spatial resolution remains an ongoing experimental challenge.

Typical techniques employed to measure cross-link density, such as solvent uptake or measurements of changes in various mechanical properties, do not generally provide spatially resolved information but rather

\footnotetext{
* To whom correspondence should be addressed.

† Analytical Sciences Laboratory, The Dow Chemical Company.

‡ Superabsorbent Products R\&D, The Dow Chemical Company.

$\S$ North Carolina State University.

$\perp$ McMaster University.

\# Present address: Department of Chemistry, Univ. Saskatchewan, Saskatoon, SK, S7N 5C9 Canada.
}

bulk averages. ${ }^{5-9}$ Methods to characterize spatial variations in the modulus of a polymer with resolution down to $50 \mu \mathrm{m}$ have been developed. ${ }^{10,11}$ I $\mathrm{n}$ principle, atomic force microscopy (AFM) should be capable of detecting variations in cross-linking for rubbery materials or solvent swollen polymers by sensing variations in the hardness down to nanometer spatial dimensions. ${ }^{12,13}$ However, in practice, using AF M for characterizing core/ shell structure or rapidly changing cross-link density is difficult because sample shape-dependent edge effects tend to mask the effects of cross-link density changes, and many relevant materials (such as the examples discussed below) are just too soft for AFM measure ments. ${ }^{14}$ An additional difficulty is the quantification of the AFM signal. The use of nuclear magnetic resonance (NMR) chemical shift of the solvent to detect polymer swelling and thus cross-link density has been demonstrated. ${ }^{15}$ However, to have a detectable chemical shift, there must be some interaction between the solvent and the polymer, such as acid base effects or hydrogen bonding. ${ }^{15,16}$ In addition, NMR is generally not a high-resolution imaging technique; it is only capable of detecting the presence of differently cross-linked regions greater than about $20 \mu \mathrm{m}$ diameter in size. Direct imaging NMR is limited to spatial resolutions on the order of tens of microns. These characteristics of AFM and NMR limit the utility of these tools for the characterization of cross-link density gradients in gels and networks. I nhomogeneities in cross-link density in polyacrylami de gels using a combination of visible light scattering, small-angle neutron scattering, and smallangle X-ray scattering has also been described, ${ }^{17}$ but of course, these methods only provide bulk averaged information. Another method one might consider for analysis of heterogeneity in cross-link structure in hydrogels is cryofracture techniques coupled with transmission electron microscopy (TEM). The sample preparation for this would be critical to success. Since the differential in electron transmission between polymer and water is low in TEM, most likely one would need to freeze-dry the specimen to allow the effect of cross- 
Scheme 1. SAP Chemistry

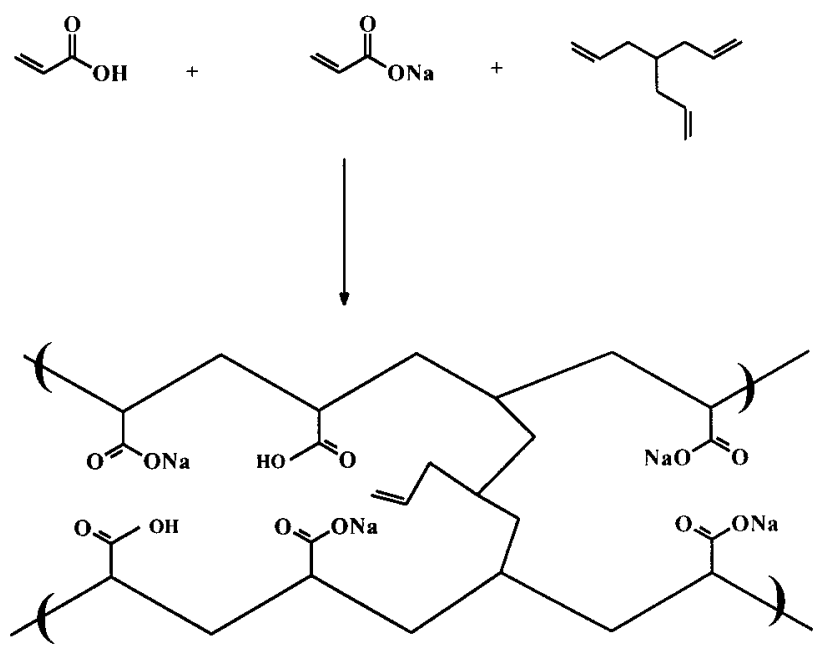

linking to be visualized. Freeze fracture techniques would be more difficult than the method discussed in the present paper, and even if one were successful in visualizing the cross-linking structure using TEM, there would be no clear method to obtain quantitative crosslinking information from such a technique.

In this paper, we describe a method to quantify the cross-link density of polymer gels at the submicron level using transmission near-edge X-ray absorption fine structure (NEXAFS) microscopy. ${ }^{18}$ To illustrate the method, we present results for the microscopic characterization of the swelling of nonuniformly cross-linked superabsorbent polymers (SAP) in sal ine solution. An abrupt, sharp change in cross-link density was detected in one material while a smooth gradient in cross-link density was observed for materials produced by a different process.

SAPs, which are a class of cross-linked polymers widely used as gels in a variety of fields, provide suitable model systems to demonstrate cross-link density characterization at the micron scale with NEXAFS microscopy. SAPs are highly engineered materials designed to simultaneously provide high fluid absorption capability, retain the fluid in a weight bearing mode with high gel strength, provide attrition resistance during manufacture, be compatible with other manufacturing materials, and retain properties necessary for hygienic uses. $3,19,20$ SAPs are made from partially neutralized poly(acrylic acid) which is lightly cross-linked throughout small $(<0.5 \mathrm{~mm})$ beads to form an insoluble, hydrophilic gel. The basic chemistry is illustrated in Scheme 1. Several different methods have been developed for surface cross-linking SAP gels as a means to improve materials properties. ${ }^{21,22}$ One way of forming the shell is to add a cross-linking agent to almost dry SAP particles al ong with enough water to swell only the outer layer of the particle surface. The typical crosslinking agent is a multifunctional molecule that undergoes a thermally induced reaction with the carboxylate groups of the polyacrylate. Three examples of reagents which have been used for cross-linking agents are included in Scheme 2. The actual morphology of the shell then will depend on the amount of surface crosslinker and the relative rates of reaction and diffusion into the solvent-swollen surface of the particle. In some cases, a cross-link gradient is expected as the amount of surface cross-linker becomes depleted by reaction coincident with diffusion of the cross-linker into the
Scheme 2. Chemical Structures of Common Cross-Linking Agents Used for Superabsorbent Polymers ${ }^{\mathrm{a}}$

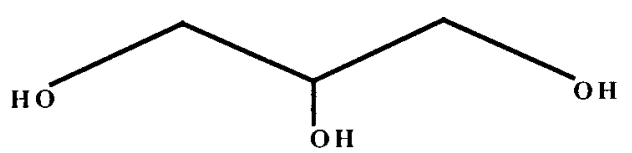

Glycerol<smiles>C(COCC1CO1)OCC1CO1</smiles>

Ethylene glycol diglycidyl ether

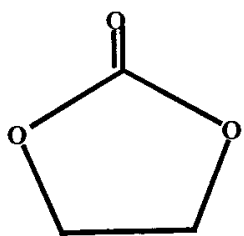

Ethylene carbonate

a Note that the chemical functionality of these materials is similar to that already present in the base polymer, thus exacerbating any chemical method of determining the presence of cross-linker directly.

particle. However, if the diffusion occurs much faster than the reaction, the cross-linker will be uniformly distributed within the solvent-swollen layer before it reacts. In such cases one might expect to find a relatively abrupt interface between the cross-linked shell and the core. Until now, the outcome of these different surface cross-link processes and the resulting core/shell structure and cross-link density gradient could not be directly visualized and assessed.

\section{Experimental Section}

Thin sections of various core-shell structured SAPs were examined in their fully hydrated state in excess $0.9 \%$ saline solution with NEXAFS microscopy. The surface of the beads was cross-linked with varying amounts of ethylene glycol diglycidyl ether or glycerol. The SAP beads created are hard and brittle when dry and extremely soft when wet. NEXAFS microscopy requires that only thin layers of the gel be examined. A two-step sample preparation protocol was developed in which sectioning and swelling are separate steps. The SAP beads were glued to an aluminum stub using epoxy, being careful that the epoxy did not wick up onto the part of the bead that was cut. Thin sections were then microtomed to about $500 \mathrm{~nm}$ in thickness. ${ }^{23}$ We were careful not to embed the beads or all ow glue to wick onto the circumference of the thin sections, as the glue would appear to be cross-linked polymer, and embedded sections may not swell freely. When there was concern, the absence of unwanted glue at the circumference was confirmed with microspectroscopy. Sections from the beads were placed on top of a $100 \mathrm{~nm}$ thick $\mathrm{Si}_{3} \mathrm{~N}_{4}$ membrane having four panes, each $1 \times 1 \mathrm{~mm}$ in size and separated by a $200 \mu \mathrm{m}$ wide supporting Si cross. ${ }^{24}$ These four fragile membranes are held rigid by a $300 \mu \mathrm{m}$ thick Si frame $5 \times 5 \mathrm{~mm}$ in overall size to provide sufficient strength for handling and mounting. After swelling of an SAP section with $0.5 \mu \mathrm{L}$ of $0.9 \%$ saline solution (an excess to fully swell the SAP), a second $\mathrm{Si}_{3} \mathrm{~N}_{4}$ window was placed on top, the two windows were gently squeezed together, and the sandwich was seal ed with silicone vacuum grease. ${ }^{25}$ This X-ray transparent wet cell was sufficiently sealed to maintain the sample hydrated for several hours during measurements. During swelling, the lateral expansion of the polymer results in a reduction in the equivalent carbon thickness (to about $50 \mathrm{~nm}$ in some areas), which is neverthel ess sufficient to be detectable with NEXAFS microscopy even in several micrometers of excess water. 


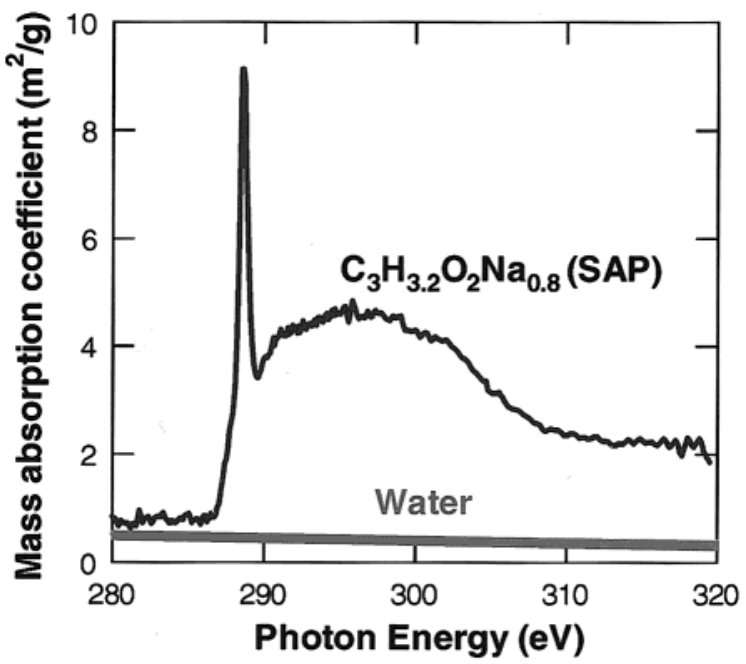

Figure 1. Mass absorption coefficient of SAP compared to that of water in the energy region of carbon 1s excitations. The large difference in cross section at $288.8 \mathrm{eV}$ is a result of the sharp $C \operatorname{ls}(C=0) \rightarrow \pi^{*}$ excitation of the carbonyl group in the acrylic acid. This NEXAFS feature provides enhanced sensitivity for the dilute polymer in a swollen, hydrated SAP.

The microscopically structured edges of our model gels were examined with the scanning transmission X-ray microscope (STXM) at beamline 7.0 at the Advanced Light Source in Berkeley, which has been described elsewhere. ${ }^{26}$ Briefly, this instrument utilizes F resnel zone plate technology to focus the $X$-ray beam to a spot, nominally $45 \mathrm{~nm}$ in diameter. The sample is scanned across the beam using stepping motor and piezoelectric driven stages, and the transmitted intensity is detected by down-converting the photons to visible light and counting photons with a photomultiplier tube. During these experiments, the spatial resolution was limited to about 100 $\mathrm{nm}$ fwhm due to vibrations in the scanning stages and the monochromator. The experiments were performed with a nominal monochromator energy resolution of $\mathrm{E} / \Delta \mathrm{E}=3000$. Visible light microscopy (VLM) provided some guidance re garding the location of the cross-linked shell in the wet cell sandwich, but with VLM only, it was not possible to distinguish differences in cross-linking.

\section{Results and Discussion}

Absorption Cross Sections and Visualization of the Cross-Link Density. The absorption cross sections of water and polymer are compared in Figure 1. The polymer spectrum was measured in absorption on the STXM microscope and cal ibrated by fitting the preedge and postedge regions to the calculated absorption coefficient. The presence of the sodium in the polymer increases the preedge absorption intensity for the polymer to a value slightly more than water. For our quantitative measurements discussed below, we acquired images at energies of 280, 289, and $320 \mathrm{eV}$. For visualizing differences in polymer density and thus cross-link density, images at $289 \mathrm{eV}$ (the most intense polymer peak) or at any energy between 289 and about $320 \mathrm{eV}$ are convenient, since the polymer absorbs more strongly than water. In these measurements of the swollen polymer, the regions with higher cross-linking density have a higher polymer density in an inverse relationship to the swelling ratio. I mages taken below the polymer C 1s absorption edge (280 eV) tend to highlight any changes in water thickness across the windows. This is true because the rel ative cross section for water and polymer are similar and in most cases, in our experiments, the apparent water thickness was about 20 times larger than that of the polymer.

In Figure 2, the STXM images at the three different $X$-ray energies are displayed along with a derived map

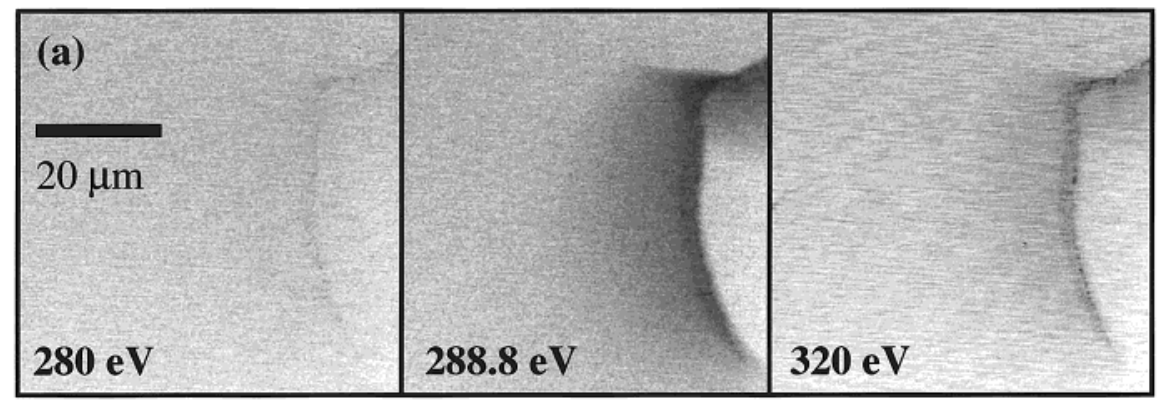

(b)

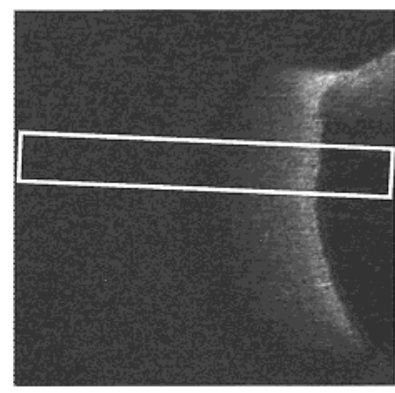

(c)

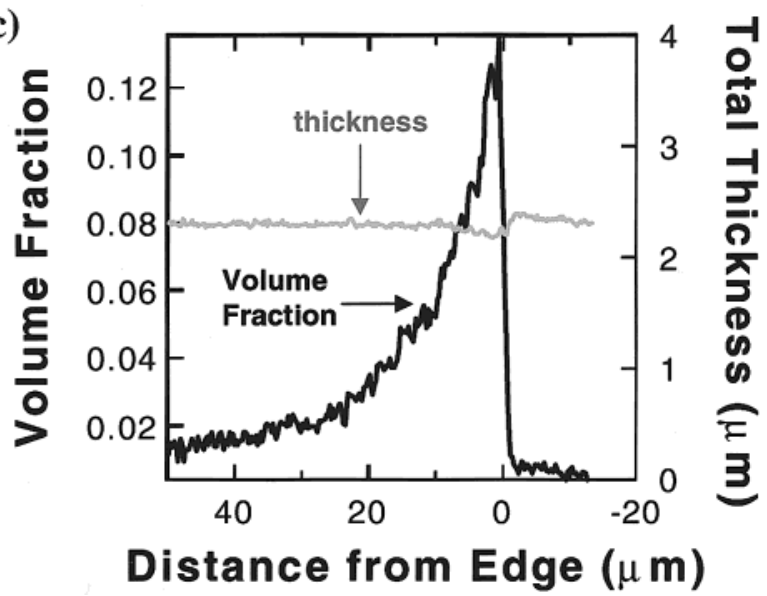

Figure 2. (A) X-ray images (transmission) of a saline-swollen section of an SAP bead which was surface cross-linked with ethylene glycol diglycidyl ether. The images were recorded at 280, 288.8, and $320 \mathrm{eV}$, as indicated. (B) Quantitative map of the polymer vol ume fraction derived via SVD from the three images. (C) Profiles of the polymer volume fraction and the total sample thickness (water and polymer) across the edge of the SAP bead, derived from the SVD analysis in the indicated region. 
(a)

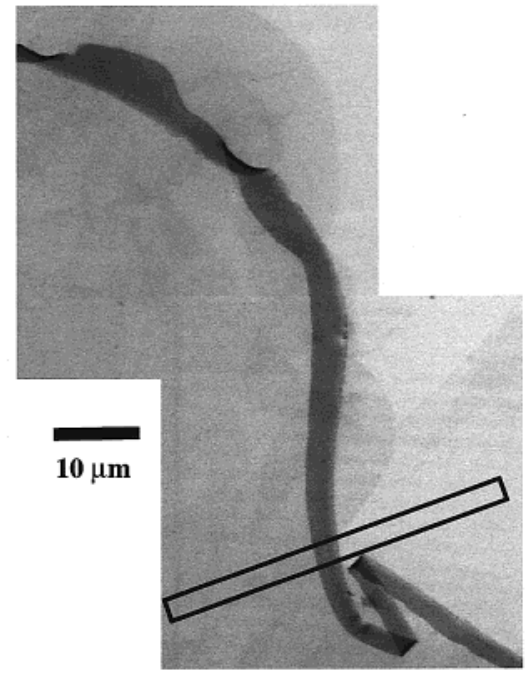

(b)

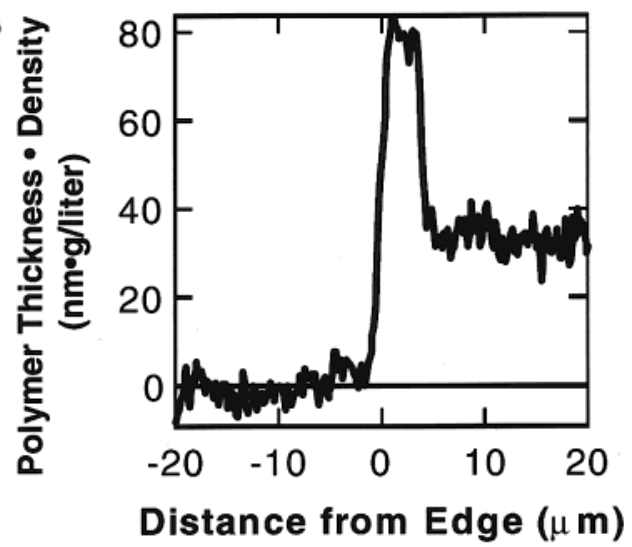

Figure 3. (A) Transmission NEXAFS image acquired at 288.8 $\mathrm{eV}$ of the mi crostructured shell of an SAP bead, surface crosslinked with glycerol. A narrow, $5 \mu \mathrm{m}$ wide band of uniform cross-link density that is twisting back and forth can be observed. (B) Profile of polymer density-thickness across the edge of the cross-linked shell in the region indicated on the image.

of the polymer volume fraction (in the gel phase only) and a plot of the total thickness and the polymer volume fraction as a function of distance parallel to a line through the shell. Images at all three energies were processed with a singular value decomposition (SVD) procedure $\mathrm{e}^{27}$ to extract quantitative polymer and water thicknesses. This method properly accounts for any gradient in the total water thickness. From these images, one can determine the relative polymer density as a function of distance from the polymer surface. In this sample the total thickness of the gel plus excess water is more than $2.0 \mu \mathrm{m}$, typical for these measurements.

Figure 3 presents transmission images of an SAP bead that is surface cross-linked with a different methodology from the SAP shown in Figure 2. The profile through the edge plotted in Figure $3 \mathrm{~b}$ is in units of thickness times density. Pronounced differences in the morphology of the shells of the two different SAPs are readily noted. The SAP in Figure 2 exhibits a gradual decay of the polymer density away from the edge over about $18 \mu \mathrm{m}$, while that in Figure 3 has an approximately $5 \mu \mathrm{m}$ wide, sharply delineated band of uniform polymer density. We note that the amount of expansion in the thin SAP section during swelling is essentially unrestricted by external constraints and

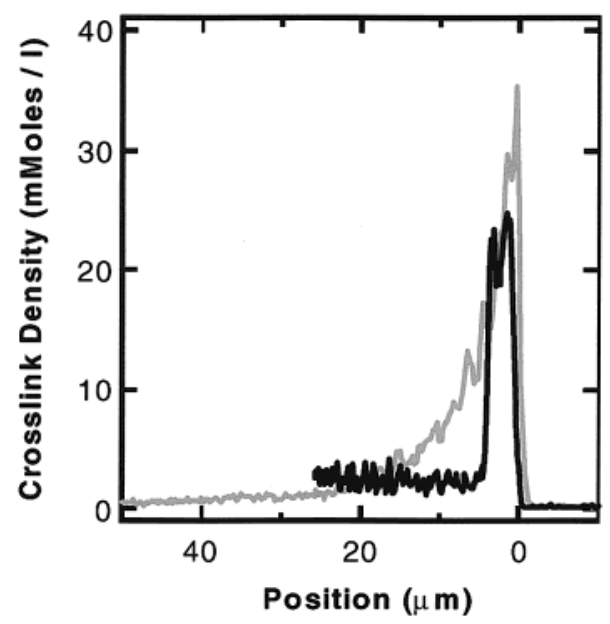

Figure 4. Comparison of the cross-link density across the shell of the SAP beads shown in Figures 2 and 3 plotted on a common quantitative scale. These profiles are derived from the regions indicated in Figures 2 and 3.

depends solely on the cross-link density. The mismatch in swelling of the shell and the core often resulted in the wrinkling or twisting of the more highly crosslinked, and hence less expanding, shell. Instances of twisting can be observed in the top right of Figure 2 . The twisting of the shell could typically be followed with the X-ray microscope over a large fraction of a millimeter and provided a convenient identification that indeed the shell was examined.

Quantitative Cross-Link Density. Quantitative cross-link density profiles across the edges of the two different SAP beads are compared in Figure 4 on a common cross-link density scale. These densities were derived from SVD-generated polymer density-thickness maps according to the foll owing procedure:

The optical density (OD) measured by STXM at any pixel will be the sum of the ODs contributed by the polymer in the gel, the water in the gel and the excess water (or saline). We assume that the swelling of the polymer by the saline solution has no discernible effect on the absorption coefficient for the polymer, but only changes the polymer density. Similar considerations apply for the water and we assume that whether the water is in the gel or external to it, it is insignificant to the absorption coefficient. Thus

$$
\mathrm{OD}=\mu_{\mathrm{p}} \mathrm{C}_{\mathrm{p}} \mathrm{t}_{\mathrm{g}}+\mu_{\mathrm{w}} \mathrm{C}_{\mathrm{w}} \mathrm{t}_{\mathrm{g}}+\mu_{\mathrm{w}} \mathrm{d}_{\mathrm{w}}\left(T-\mathrm{t}_{\mathrm{g}}\right)
$$

where $\mu_{\mathrm{p}}$ and $\mu_{\mathrm{w}}$ are the X-ray mass absorption coefficients for polymer and water (saline), respectively, $C_{p}$ and $\mathrm{C}_{\mathrm{w}}$ are the concentrations of polymer and water in the gel, $d_{w}$ is the density of neat sal ine $\left(1.01 \mathrm{~g} / \mathrm{cm}^{3}\right), t_{g}$ is the thickness of the swollen gel, and T is the total cell (gel plus the free saline) thickness.

When the polymer is swollen, the STXM measurement is only sensitive to the lateral expansion of the polymer, which decreases the areal density of polymer. Changes in density in the direction parallel to the X-ray beam are not discernible. The concentration of polymer, water, and the gel thickness can all be expressed in terms of the volume fraction of polymer $\mathrm{v}_{2}$ :

$$
C_{p}=v_{2} d_{p} ; \quad C_{w}=\left(1-v_{2}\right) d_{w} ; \quad t_{g}=t_{0} / v_{2}^{1 / 3}
$$

where $d_{p}$ is the density of the dry polymer $\left(1.66 \mathrm{~g} / \mathrm{cm}^{3}\right)$ and $t_{0}$ is the thickness of the section before swelling. 
The initial dry polymer thickness is presumed constant across the entire thin section, but the actual thickness was generally only estimated from the microtome setting and may differ from this value according to the extent of hydration caused by ambient humidity during sample preparation. Substituting these relationships into eq 1 gives

$$
\begin{aligned}
\mathrm{OD} & =\mu_{\mathrm{p}} \mathrm{v}_{2} \mathrm{~d}_{\mathrm{p}} \mathrm{t}_{0} / \mathrm{v}_{2}^{1 / 3}+\mu_{\mathrm{w}}\left(1-\mathrm{v}_{2}\right) \mathrm{d}_{\mathrm{w}} \mathrm{t}_{0} / \mathrm{v}_{2}{ }^{1 / 3}+ \\
& \mu_{\mathrm{w}} \mathrm{d}_{\mathrm{w}}\left(\mathrm{T}-\mathrm{t}_{0} / \mathrm{v}_{2}{ }^{1 / 3}\right) \\
& =\mu_{\mathrm{p}} \mathrm{d}_{\mathrm{p}} \mathrm{t}_{0} \mathrm{v}_{2}^{2 / 3}-\mu_{\mathrm{w}} \mathrm{d}_{\mathrm{w}} \mathrm{t}_{0} / \mathrm{v}^{2 / 3}+\mu_{\mathrm{w}} \mathrm{d}_{\mathrm{w}} \mathrm{T} \\
& =\mu_{\mathrm{p}}\left(1.66 \mathrm{t}_{0} \mathrm{v}_{2}^{2 / 3}\right)+\mu_{\mathrm{w}}\left(\mathrm{T}-\mathrm{t}_{0} \mathrm{v}_{2}{ }^{2 / 3}\right)
\end{aligned}
$$

Set

$$
1.66 \mathrm{t}_{0} \mathrm{v}_{2}^{2 / 3}=\tau_{\mathrm{p}} \text { and } \mathrm{T}-\mathrm{t}_{0} \mathrm{v}_{2}^{2 / 3}=\tau_{\mathrm{w}}
$$

Then

$$
\mathrm{v}_{2}=\left(\tau_{\mathrm{p}} / 1.66 \mathrm{t}_{0}\right)^{3 / 2}
$$

Starting from the Flory-Rehner equation, ${ }^{5}$ one can derive the following

$$
X L=-v_{2}{ }^{1 / 3}\left[\ln \left(1-v_{2}\right)+v_{2}+\chi v_{2}^{2}\right] / N_{1}
$$

where $\mathrm{XL}$ is the relative cross-link density, $\chi$ is the Flory-Huggins interaction parameter (0.469), $\mathrm{V}_{1}$ is the molar volume of water $(0.018 \mathrm{~L} / \mathrm{mol})$, and $v_{2}$ is the volume fraction of polymer in the swollen polymer phase.

The large differences between the two kinds of SAPs are readily observable in Figure 4, including sharp transitions on the micron or submicron length scale. The shapes of the cross-link density profiles reveal information on the kinetics of the process chemistry. The structure of the shell is determined by a complicated interplay between the dynamics of swelling of the bead in water, the diffusion rate of the cross-linker in the water phase (if the cross-linker is added in a separate step), and the rate of the cross-linking reaction. TheSAP sample examined in Figure 2 shows a gradual crosslinking gradient. In contrast, the SAP examined in Figure 3 has an abrupt internal boundary between the shell and core. The visualization and quantitative measurement of the cross-link density profile are important to understanding the reaction dynamics in detail.

\section{Conclusions}

In summary, we have used NEXAFS microscopy to quantify the cross-link density of swollen gels at the submicron level. Two extreme cross-link concentration profiles were observed in surface cross-linked SAPs that are the result of differences in the kinetics of the chemical reaction that created the core-shell structure in the materials examined. These results show there is a correlation between morphology and the diffusion vs reaction rate dependence of the cross-linking reaction. The methodology and technique devel oped provide a useful tool for research and development of optimized cross-link density structures. The spatial resolution in the present results is limited by signal-to-noise and recorded pixel density. The examples investigated in the present study had feature sizes of several micrometers and thus did not burden the available spatial resolution, but modern X-ray microscopes are capable of discerning feature sizes of $30 \mathrm{~nm}$ or less. Sample feature size did not require better resolution to provide substantial understanding of the cross-link density gradients here. The methodol ogy is extendable to any other swollen gel system for which the solvent and polymer network have distinct NEXAFS features at an appropriate core absorption edge. Typically, good NEXAFS contrast between materials is the rule rather than exception. The presented results should thus be of interest in a range of disciplines, such as biology, medicine, cosmetics research, agriculture, and even food science. ${ }^{28}$

Acknowledgment. The ALS STXM was developed by T. Warwick (ALS), B. Tonner (UWM), and collaborators, with support from the U.S. DOE under Contract DE-AC03-76SF00098. Zone plates used at ALS are provided by Erik Anderson from the CXRO, LBNL. We thank Uli Neuhäusler and C. J acobsen for help with initial wet cell technology development and J. VanZanten for critical comments. I nitial work on this project was performed using the Stony Brook STXM at beamline X1A at the NSLS. This work was supported in part (H. Ade) by NSF NYI award (DMR-9458060) and by an NSERC strategic grant (A. Hitchcock).

\section{References and Notes}

(1) DeRossi, D., Kajiwara, K., Osada, Y., Yamauchi, A., Eds. Polymer Gels: Fundamentals and Biomedical Applications; Plemum Press: New York, 1991.

(2) Addad, J. P. C., Ed.; Physical Properties of Polymeric Gels; J ohn Wiley \& Sons: New York, 1996

(3) Buchholz, F. L. In Modern Superabsorbent Polymer Technol ogy; Buchholz, F. L., Graham, A. T., Eds.; Wiley-VCH: New York, 1998; pp 251-272.

(4) Funke, W. Plast., Rubber Process. Appl. 1983, 3, 243-247.

(5) Flory, P.; Rehner, J . J . Phys. Chem. 1943, 11, 512-520.

(6) Flory, P.; Rehner, J . J . Phys. Chem. 1943, 11, 521-526.

(7) Erede, L. Macromol ecules 1986, 19, 654-658.

(8) Sen, M.; Yakar, A.; Guven, O. Polymer 1999, 40, 2969-2974.

(9) Vera-Graziano, R.; Hernandez-Sanchez, F.; Cauich-Rodriguez, J . J . Appl. Polym. Sci. 1995, 55, 1317-1327.

(10) Gillen, K.; Clough, R.; Quintana, C. Polym. Degrad. Stab. 1987, 17, 31-47.

(11) Gillen, K.; Clough, R. Polym. Eng. Sci. 1989, 29, 29-35.

(12) Bar, G.; Delineau, L.; Brandisch, R.; Bruch, M.; Whangbo, M. H. Appl. Phys. Lett. 1999, 4198-4200.

(13) Mareanukroh, M.; Hamed, G.; Eby, R. Rubber Chem. Technol. 1996, 69, 801-806.

(14) Meyers, G., unpublished results.

(15) O'Connor, P.; Cutie, S.; Smith, P.; Martin, S.; Sammler, R.; Harris, W.; Marks, M.; Wilson, L. Macromolecules 1996, 29, $7872-7884$

(16) Ogino, K.; Sato, H. J . Appl. Polym. Sci. 1995, 58, 1015-1020.

(17) Hecht, A.-M.; Duplessix, R.; Geissler, E. Macromolecules 1985, 18, 2167-2173.

(18) Ade, H.; Zhang, X.; Cameron, S.; Costello, C.; Kirz, J .; Williams, S. Science 1992, 258, 972-975.

(19) Shimomura, T.; Namba, T. In Superabsorbent Polymers Scienceand Technol ogy; Buchholz, F. L., Peppas, N. A., Eds.; American Chemical Society: Washington, DC, 1994; pp 112127.

(20) Hogari, K.; Ashiya, F. In Superabsorbent Polymers Science and Technology; Buchholz, F. L., Peppas, N. A., Eds.; American Chemical Society: Washington, DC, 1994; pp 12840.

(21) Staples, T. L.; Henton, D. E.; Buchholz, F. L. In Modern Superabsorbent Polymer Technology; Buchholz, F. L., Graham, A. T., Eds.; Wiley-VCH: New York, 1998; pp 55-60.

(22) Buchholz, F. L. In Modern Superabsorbent Polymer Technology; Buchholz, F. L., Graham, A. T., Eds.; Wiley-VCH: New York, 1998; pp 190-3.

(23) Microtoming required careful control of the state of hydration of the bead. When the air was too humid, the beads needed to be partially dried in an oven $\left(60^{\circ} \mathrm{C}\right)$ prior to microtoming. 
(24) Silson Ltd, J BJ Business Park, Northampton Road, Blisworth, Northampton England, NN7 3DW

(25) Related wet cell technology has been developed and used by Neuhäusler et al. [Neuhäusler, U.;] acobsen, C.: Schulze, D. Stott, D.; Abend, S. J . Synchrotron Radiat. 2000, 7, 110112] for environmental studies. $\mathrm{Si}_{3} \mathrm{~N}_{4}$ technology for wet cells has been pioneered by Rothmann et al. in 1986 to study zymogen granules.

(26) Warwick, T.; Franck, T.; Kortright, J .; Meigs, G.; Moronne,
M.; Myneni, S.; Rotenberg, E.; Seal, S.; Steele, W.; Ade, H.; Garcia, A.; Cerasari, S.; Denlinger, J .; Hayakawa, S.; Hitchcock, A.; Tyliszczak, T.; Rightor, E.; Shin, H.-J .; Tonner, B. Rev. Sci. Instrum. 1998, 69, 2964-2973.

(27) Zhang, X.; Balhorn, R.; Mazrimas, J .; Kirz, J . J . Struct. Biol. 1996, 116, 335-344.

(28) Hermansson, A. M.; Langton, M.; Loren, N. MRS Bull. 2000, $25,30-37$

MA010840D 